PROCEEDINGS OF THE

AMERICAN MATHEMATICAL SOCIETY

Volume 125, Number 6, June 1997, Pages 1625-1634

S 0002-9939(97)03781-7

\title{
GENERALIZED NUMERICAL RANGES, JOINT POSITIVE DEFINITENESS AND MULTIPLE EIGENVALUES
}

\author{
YIU TUNG POON
}

(Communicated by Palle E. T. Jorgensen)

Dedicated to Professor Yik Hoi Au-Yeung

\begin{abstract}
We prove a convexity theorem on a generalized numerical range that combines and generalizes the following results: 1) Friedland and Loewy's result on the existence of a nonzero matrix with multiple first eigenvalue in subspaces of hermitian matrices, 2) Bohnenblust's result on joint positive definiteness of hermitian matrices, 3) the Toeplitz-Hausdorff Theorem on the convexity of the classical numerical range and its various generalizations by Au-Yeung, Berger, Brickman, Halmos, Poon, Tsing and Westwick.
\end{abstract}

\section{INTRODUCTION}

The study of hermitian matrices arises naturally in many branches of mathematics. (See $[\mathrm{HJ}]$ for a nice introduction on the subject.) We are going to study the following areas of hermitian matrices: multiplicity of the largest eigenvalue, positive definiteness, convexity and inclusion of generalized numerical ranges.

Let $A_{1}, \cdots, A_{p}$ be $p n \times n$ hermitian matrices and $\mathcal{K}$ the real linear subspace spanned by $A_{1}, \cdots, A_{p}$. There has been considerable interest in studying the joint behavior of $A_{1}, \cdots, A_{p}$. For example:

Friedland and Loewy [FL] proved that for every $1<r<n$ there exists $k(r)$ such that if $\operatorname{dim} \mathcal{K} \geq k(r)$, then there exists a non-zero matrix $A$ in $\mathcal{K}$ such that the largest eigenvalue of $A$ has multiplicity $\geq r$. (See Proposition 1.3.)

Bohnenblust $[\mathrm{Bo}]$ gave a necessary and sufficient condition for the existence of a positive definite matrix in $\mathcal{K}$. (See Proposition 1.2.)

$\mathrm{Au}$-Yeung and Poon [AP] showed that Bohnenblust's result is equivalent to a result which generalizes the Toeplitz-Hausdorff Theorem [To], [Hau] on the convexity of numerical range. (See Proposition 1.1.)

The Toeplitz-Hausdorff Theorem has been generalized in many directions by AuYeung [A1], Halmos [Hal1] and Berger [Be], Westwick [W], Au-Yeung and Tsing [AT1], [AT2]. Au-Yeung and Tsing [AT1], [AT2] have also shown that the convexity theorem of the joint c-numerical range is equivalent to some joint positive

Received by the editors September 22, 1995 and, in revised form, January 4, 1996.

1991 Mathematics Subject Classification. Primary 15A60; Secondary 47A12.

Key words and phrases. Generalized numerical range, convexity, joint positive definiteness, multiple eigenvalue.

The author wants to thank the referee for some helpful comments and suggestions.

(C)1997 American Mathematical Society 
definiteness property, which, in turn, is equivalent to some inclusion relations on the joint c-numerical ranges.

In this paper, we are going to prove a theorem, which will combine and generalize all of the results mentioned above.

Let $\mathbf{R}$ be the field of real numbers, $\mathbf{C}$ the field of complex numbers and $\mathbf{Q}$ the skew field of real quaternions. We will use $\mathbf{F}$ to denote any one of $\mathbf{R}, \mathbf{C}$ or $\mathbf{Q}$. For an $n \times m$ matrix $A$ over $\mathbf{F}, A^{*}$ will denote the conjugate transpose of $A$. For this purpose, vectors in $\mathbf{F}^{n}$ will be identified with $1 \times n$ matrices over $\mathbf{F} . \mathcal{M}_{n}(\mathbf{F})$ will denote the set of all $n \times n$ matrices over $\mathbf{F}$. For $\mathbf{c} \in \mathbf{F}^{n}$, [c] will denote the diagonal matrix in $\mathcal{M}_{n}(\mathbf{F})$ with diagonal c. Suppose $A_{i} \in \mathcal{M}_{n_{i}}(\mathbf{F}), 1 \leq i \leq m$. Then $A_{1} \oplus \cdots \oplus A_{m}$ will represent the block diagonal matrix formed by $A_{1}, \cdots, A_{m}$.

Let $\mathcal{H}_{n}(\mathbf{F})=\left\{A \in \mathcal{M}_{n}(\mathbf{F}): A=A^{*}\right\}$ and $\mathcal{U}_{n}(\mathbf{F})=\left\{A \in \mathcal{M}_{n}(\mathbf{F}): A A^{*}=I_{n}\right\}$ be the hermitian and unitary matrices in $\mathcal{M}_{n}(\mathbf{F})$ respectively. Here, $I_{n}$ denotes the $n \times n$ identity matrix. For each $n \geq 1, \mathcal{H}_{n}(\mathbf{F})$ is a real vector space of dimension $f_{\mathbf{F}}(n)$, where $f_{\mathbf{R}}(n)=n(n+1) / 2, f_{\mathbf{C}}(n)=n^{2}$, and $f_{\mathbf{Q}}(n)=n(2 n-1)$. Thus, we can identify $\mathcal{H}_{n}(\mathbf{F})$ with $\mathbf{R}^{f_{\mathbf{F}}(n)}$, equipped with the usual topology.

A subset $S$ of $\mathbf{R}^{m}$ is said to be convex if for every $\mathbf{x}, \mathbf{y} \in S$, we have $t \mathbf{x}+(1-t) \mathbf{y} \in$ $S$ for all $0 \leq t \leq 1$. Given a subset $S$ of $\mathbf{R}^{m}$, conv $S$ will denote the smallest convex subset of $\mathbf{R}^{m}$ containing $S$. A subset $P \subseteq \mathbf{R}^{m}$ is called a hyperplane if there exist a subspace $V$ of $\mathbf{R}^{m}$ and $a \in \mathbf{R}^{m}$ such that $P=\{a+v: v \in V\}$. A non-empty subset $S$ of $\mathbf{R}^{m}$ is said to have a convex boundary if there exists a hyperplane $P$ such that $S \subseteq P$ and the boundary of conv $S$, as a topological subspace of $P$, is contained in $S$.

Let $W^{\mathbf{F}}\left(A_{1}, A_{2}\right)=\left\{\left(\mathbf{x} A_{1} \mathbf{x}^{*}, \mathbf{x} A_{2} \mathbf{x}^{*}\right): \mathbf{x} \in \mathbf{F}^{n}, \mathbf{x x}^{*}=1\right\}$, for $A_{1}, A_{2} \in \mathcal{H}_{n}(\mathbf{F})$. The Toeplitz-Hausdorff Theorem [To], [Hau] states that $W^{\mathbf{C}}\left(A_{1}, A_{2}\right)$ is a convex subset of $\mathbf{R}^{2}$ for all $A_{1}, A_{2} \in \mathcal{H}_{n}(\mathbf{C})$. Brickman [Br] proved that if $n>2$, then $W^{\mathbf{R}}\left(A_{1}, A_{2}\right)$ is convex for all $A_{1}, A_{2} \in \mathcal{H}_{n}(\mathbf{R})$. Au-Yeung [A1] gave a unified proof for the convexity of $W^{\mathbf{F}}\left(A_{1}, A_{2}\right)$ for $\mathbf{F}=\mathbf{R}(n>2)$ and $\mathbf{F}=\mathbf{C}$ or $\mathbf{Q}$.

One direction of generalization of the Toeplitz-Hausdorff Theorem is to consider the set $W^{\mathbf{F}}\left(A_{1}, \cdots, A_{p}\right)=\left\{\left(\mathbf{x} A_{1} \mathbf{x}^{*}, \cdots, \mathbf{x} A_{p} \mathbf{x}^{*}\right): \mathbf{x} \in \mathbf{F}^{n}, \mathbf{x x}^{*}=1\right\}$ for $A_{1}, \cdots, A_{p} \in \mathcal{H}_{n}(\mathbf{F})$. Hausdorff [Hau] noted that $W^{\mathbf{C}}\left(A_{1}, A_{2}, A_{3}\right)$ has a convex boundary and remarked that $W^{\mathrm{C}}\left(A_{1}, A_{2}, A_{3}\right)$ is not convex in general. However, for $n \geq 3, W^{\mathbf{C}}\left(A_{1}, A_{2}, A_{3}\right)$ is convex for all $A_{1}, A_{2}, A_{3} \in \mathcal{H}_{n}(\mathbf{C})$. This is a special case of the following

Proposition 1.1. Let $\mathbf{F}=\mathbf{R}, \mathbf{C}$, or $\mathbf{Q}, 1 \leq r \leq n-1, p<f_{\mathbf{F}}(r+1)-\delta_{n, r+1}$ and $A_{1}, \cdots, A_{p} \in \mathcal{H}_{n}(\mathbf{F})$. Then the set

$$
W_{r}^{\mathbf{F}}\left(A_{1}, \cdots, A_{p}\right)=\left\{\left(\sum_{i=1}^{r} \mathbf{x}_{\mathbf{i}} A_{1} \mathbf{x}_{\mathbf{i}}^{*}, \cdots, \sum_{i=1}^{r} \mathbf{x}_{\mathbf{i}} A_{p} \mathbf{x}_{\mathbf{i}}^{*}\right): \mathbf{x}_{\mathbf{i}} \in \mathbf{F}^{n}, \sum_{i=1}^{r} \mathbf{x}_{\mathbf{i}} \mathbf{x}_{\mathbf{i}}^{*}=1\right\}
$$

is convex. Here $\delta_{i, j}$ is the Kronecker delta.

$\mathrm{Au}-$ Yeung and Poon showed [AP] that Proposition 1.1 is equivalent to the following result of Bohnenblust $[\mathrm{Bo}]$, [Ta] on joint positive definiteness of hermitian matrices. 
Proposition 1.2. Let $\mathbf{F}=\mathbf{R}, \mathbf{C}$ or $\mathbf{Q}, 1 \leq r \leq n-1, p<f_{\mathbf{F}}(r+1)-\delta_{n, r+1}$ and $A_{1}, \cdots, A_{p} \in \mathcal{H}_{n}(\mathbf{F})$. Suppose $(0, \cdots, 0) \notin W_{r}^{\mathbf{F}}\left(A_{1}, \cdots, A_{p}\right)$. Then there exist $\alpha_{1}, \cdots, \alpha_{p} \in \mathbf{R}$ such that the matrix $\sum_{i=1}^{p} \alpha_{i} A_{i}$ is positive definite.

Bohnenblust proved Proposition 1.2 for $\mathbf{F}=\mathbf{R}$ and $\mathbf{C}$ but, as noted in [AP], the result remains valid for $\mathbf{F}=\mathbf{Q}$. Friedland and Lowey $[\mathrm{FL}]$ proved the next proposition and then showed that it is also equivalent to Proposition 1.2, for $\mathbf{F}=\mathbf{R}$ or $\mathbf{C}$.

Proposition 1.3. Let $\mathbf{F}=\mathbf{R}$ or $\mathbf{C}, 1<r<n$, and $\mathbf{k}(r)=f_{\mathbf{F}}(n)-f_{\mathbf{F}}(n+1-r)$. Suppose $\mathcal{K}$ is a subspace of $\mathcal{H}_{n}(\mathbf{F})$ with dimension $\geq \mathbf{k}(r)$. Then there exists a non-zero matrix $A \in \mathcal{K}$ such that $\lambda_{1}(A)=\cdots=\lambda_{r}(A)$.

Here, for a matrix $B \in \mathcal{H}_{n}(\mathbf{F}), \lambda_{1}(B) \geq \lambda_{2}(B) \geq \cdots \geq \lambda_{n}(B)$ are the eigenvalues of $B$ arranged in decreasing order.

Remark 1.4. a) The bound $f_{\mathbf{F}}(r+1)-\delta_{n, r+1}$ in Proposition 1.1 is best possible in the sense that for every $1 \leq r \leq n-1$ and $p \geq f_{\mathbf{F}}(r+1)-\delta_{n, r+1}$, there exist $A_{1}, \cdots, A_{p} \in \mathcal{H}_{n}(\mathbf{F})$ such that $W_{r}^{\overline{\mathbf{F}}}\left(A_{1}, \cdots, A_{p}\right)$ is not convex.

b) The set $W^{\mathbf{F}}\left(A_{1}, \cdots, A_{p}\right)$ has appeared in the study of structured singular values in control theory $[\mathrm{D}],[\mathrm{FT}]$, which leads to the problem of determining the smallest $r$ such that every $\mathbf{w}$ in conv $W^{\mathbf{F}}\left(A_{1}, \cdots, A_{p}\right)$ can be expressed as a convex combination of not more than $r$ points in $W^{\mathbf{F}}\left(A_{1}, \cdots, A_{p}\right)$. It has been shown in [P2] that the inequality $p<f_{\mathbf{F}}(r+1)-\delta_{n, r+1}$ gives the best possible bound for $r$.

Let $\mathbf{c}=\left(c_{1}, \cdots, c_{n}\right) \in \mathbf{R}^{n}$, and $A_{1}, \cdots, A_{p} \in \mathcal{H}_{n}(\mathbf{F})$. To state another generalization of the Toeplitz-Hausdorff Theorem, define

$$
\begin{gathered}
\mathcal{O}_{n}(\mathbf{F})=\left\{\left\{\mathbf{x}_{\mathbf{1}}, \cdots, \mathbf{x}_{\mathbf{n}}\right\}: \mathbf{x}_{\mathbf{i}} \in \mathbf{F}^{n}, \mathbf{x}_{\mathbf{i}} \mathbf{x}_{\mathbf{j}}^{*}=\delta_{i j}\right\}, \\
W_{\mathbf{c}}^{\mathbf{F}}\left(A_{1}, \cdots, A_{p}\right) \\
=\left\{\left(\sum_{i=1}^{n} c_{i} \mathbf{x}_{\mathbf{i}} A_{1} \mathbf{x}_{\mathbf{i}}^{*}, \cdots, \sum_{i=1}^{n} c_{i} \mathbf{x}_{\mathbf{i}} A_{p} \mathbf{x}_{\mathbf{i}}^{*}\right):\left\{\mathbf{x}_{\mathbf{1}}, \cdots, \mathbf{x}_{\mathbf{n}}\right\} \in \mathcal{O}_{n}(\mathbf{F})\right\} .
\end{gathered}
$$

Let $1 \leq k \leq n$. Halmos [Hal1] conjectured that if $\mathbf{c}$ has $k$ coordinates equal to 1 and the rest 0 , then $W_{\mathbf{c}}^{\mathbf{C}}\left(A_{1}, A_{2}\right)$ is convex. The conjecture was proven by Berger [Be], [Hal2]. Berger's result was generalized by Westwick [W], who proved the following result for $\mathbf{F}=\mathbf{C}$. A simple proof of Westwick's result is given in [P1]. The following proposition was proven by Au-Yeung and Tsing [AT1].

Proposition 1.5. Let $\mathbf{c} \in \mathbf{R}^{n}$. If $F=\mathbf{R}$ and $n>2$ or $\mathbf{F}=\mathbf{C}$, $\mathbf{Q}$, then $W_{\mathbf{c}}^{\mathbf{F}}\left(A_{1}, A_{2}\right)$ is convex for all $A_{1}, A_{2} \in \mathcal{H}_{n}(\mathbf{F})$.

$\mathrm{Au}$-Yeung [A2] has called for a unified proof of Propositions 1.1 and 1.5. The following result of $\mathrm{Au}$-Yeung and Tsing [AT2] is the first step in this direction.

Proposition 1.6. Let $n \geq 3, \mathbf{c} \in \mathbf{R}^{n}$. Then $W_{\mathbf{c}}^{\mathbf{C}}\left(A_{1}, A_{2}, A_{3}\right)$ is convex for all $A_{1}, A_{2}, A_{3} \in \mathcal{H}_{n}(\mathbf{C})$.

We note that for $\mathbf{c}=(1,0, \cdots, 0) \in \mathbf{R}^{n}, W_{\mathbf{c}}^{\mathbf{C}}\left(A_{1}, A_{2}, A_{3}\right)=W_{1}^{\mathbf{C}}\left(A_{1}, A_{2}, A_{3}\right)$. Thus, for $n \geq 3$ and $\mathbf{F}=\mathbf{C}$, Proposition 1.6 covers both Proposition 1.5 and the case when $r=1$ in Proposition 1.1.

In this paper, we will prove a result which covers Propositions 1.1, 1.2, 1.31 .5 and 1.6 . 


\section{MAIN THEOREM}

A square matrix with nonnegative entries is said to be doubly stochastic (d.s.) if every row sum and every column sum is equal to 1 . Let $\Omega(n)$ be the set of all $n \times n$ d.s. matrices and $\Omega(n, r)=\left\{\oplus_{i=1}^{m} D_{i}: m \geq 1, D_{i} \in \Omega\left(n_{i}\right), n_{i} \leq r, \sum_{i=1}^{m} n_{i}=n\right\}$. We have $\Omega(n, 1) \subseteq \Omega(n, 2) \subseteq \cdots \subseteq \Omega(n, n)=\Omega(n)$.

For $\mathbf{c}=\left(c_{1}, \cdots, c_{n}\right)$, let $c_{[1]} \geq \cdots \geq c_{[n]}$ be the decreasing rearrangement of the components of $\mathbf{c}$ and $\mathbf{c}_{\downarrow}=\left(c_{[1]}, \cdots, c_{[n]}\right)$.

Definition 2.1. Let $\mathbf{c} \in \mathbf{R}^{n}, \mathbf{F}=\mathbf{R}, \mathbf{C}$ or $\mathbf{Q}$ and $A_{1}, \cdots, A_{p} \in \mathcal{H}_{n}(\mathbf{F})$. For $1 \leq r \leq n$, define

$$
\begin{aligned}
\mathcal{C}(\mathbf{c}, r) & =\left\{\mathbf{c}_{\downarrow} D: D \in \Omega(n, r)\right\}, \text { and } \\
W_{\mathbf{c}, r}^{\mathbf{F}}\left(A_{1}, \cdots, A_{p}\right) & =\left\{\left(\operatorname{tr}[\mathbf{b}] U A_{1} U^{*}, \cdots, \operatorname{tr}[\mathbf{b}] U A_{p} U^{*}\right): \mathbf{b} \in \mathcal{C}(\mathbf{c}, r), U \in \mathcal{U}_{n}(\mathbf{F})\right\},
\end{aligned}
$$

where $\operatorname{tr} A$ denotes the trace of $A$.

The main result in this paper is

Theorem 2.2. Let $\mathbf{c} \in \mathbf{R}^{n}, \mathbf{F}=\mathbf{R}$, $\mathbf{C}$ or $\mathbf{Q}, 1 \leq r \leq n-1, p<f_{\mathbf{F}}(r+1)-\delta_{n, r+1}$ and $A_{1}, \cdots, A_{p} \in \mathcal{H}_{n}(\mathbf{F})$. Then $W_{\mathbf{c}, r}^{\mathbf{F}}\left(A_{1}, \cdots, A_{p}\right)$ is convex.

Remark 2.3. We note that for $\mathbf{e}=(1,0, \cdots, 0)$ and $\mathbf{c} \in \mathbf{R}^{n}$, we have

$$
\begin{aligned}
& W_{\mathbf{e}, r}^{\mathbf{F}}\left(A_{1}, \cdots, A_{p}\right)=W_{r}^{\mathbf{F}}\left(A_{1}, \cdots, A_{p}\right), \text { and } \\
& W_{\mathbf{c}, 1}^{\mathbf{F}}\left(A_{1}, \cdots, A_{p}\right)=W_{\mathbf{c}}^{\mathbf{F}}\left(A_{1}, \cdots, A_{p}\right) .
\end{aligned}
$$

For $r=1, f_{\mathbf{F}}(r+1)-\delta_{n, r+1}>\left\{\begin{array}{ll}2 & \text { if } \mathbf{F}=\mathbf{R} \text { and } n \geq 3 \text { or } \mathbf{F}=\mathbf{C}, \mathbf{Q} \\ 3 & \text { if } n \geq 3 \text { and } \mathbf{F}=\mathbf{C}, \mathbf{Q}\end{array}\right.$. Therefore, Theorem 2.2 is a generalization of Propositions 1.1, 1.5 and 1.6.

For the rest of this section, $\mathbf{F}=\mathbf{R}, \mathbf{C}$ or $\mathbf{Q}$. If $\mathbf{d}^{i} \in \mathbf{R}^{n_{i}}$, for $1 \leq i \leq m$ with $\sum_{i=1}^{m} n_{i}=n$, then $\left(\mathbf{d}^{1}, \mathbf{d}^{2}, \cdots, \mathbf{d}^{m}\right)$ will denote the vector in $\mathbf{R}^{n}$ formed by the juxtaposition of the components in $\mathbf{d}^{i}$.

Lemma 2.4. a) Let $A \in \mathcal{M}_{n}(\mathbf{F})$. Then $A \in \mathcal{H}_{n}(\mathbf{F})$ if and only if there exist $U \in \mathcal{U}_{n}(\mathbf{F})$ and $\mathbf{c} \in \mathbf{R}^{n}$ such that $A=U[\mathbf{c}] U^{*}$.

b) Let $U \in \mathcal{U}_{n}(\mathbf{R})$. There is a path in $\mathcal{U}_{n}(\mathbf{R})$ connecting $U$ to $I_{n}$ if and only if $\operatorname{det} U=1$. For $\mathbf{F}=\mathbf{C}$ or $\mathbf{Q}, \mathcal{U}_{n}(\mathbf{F})$ is path connected.

Proof. For $\mathbf{F}=\mathbf{R}$ or $\mathbf{C}$, the result is well known $[\mathrm{HJ}]$. For $\mathbf{F}=\mathbf{Q}$, a) and b) follow from Theorem 9 and 8 of Lee [Le] respectively.

For $A \in \mathcal{M}_{n}(\mathbf{F})$, let $\operatorname{Tr} A=\operatorname{Re} \operatorname{tr} A$, the real part of $\operatorname{tr} A$. Then we have

Lemma 2.5. For $A, B \in \mathcal{M}_{n}(\mathbf{F}),\langle A, B\rangle=\operatorname{Tr} A B^{*}$ defines a real valued inner product on $\mathcal{M}_{n}(\mathbf{F})$. Moreover, $\operatorname{Tr} A B=\operatorname{Tr} B A$ for all $A, B \in \mathcal{M}_{n}(\mathbf{F})$.

Proof. Since the result is well known for $\mathbf{F}=\mathbf{R}$ or $\mathbf{C}$, it remains to prove the case when $\mathbf{F}=\mathbf{Q}$. Recall that $\mathbf{Q}$ is generated by $\mathbf{C}$ and $\mathbf{j}$ such that $a \mathbf{j}=\mathbf{j} \bar{a}$ for all $a \in \mathbf{C}$. Thus, every $A \in \mathcal{M}_{n}(\mathbf{Q})$ can be expressed uniquely as $A=A_{1}+\mathbf{j} A_{2}$, with $A_{1}, A_{2} \in \mathcal{M}_{n}(\mathbf{C})$. This induces (see Lee [Le]) a unital real algebra isomorphism $f$ of $\mathcal{M}_{n}(\mathbf{Q})$ into a subalgebra of $\mathcal{M}_{2 n}(\mathbf{C})$ by

$$
f\left(A_{1}+\mathbf{j} A_{2}\right)=\left[\begin{array}{ll}
A_{1} & -\overline{A_{2}} \\
A_{2} & \overline{A_{1}}
\end{array}\right] .
$$


Here, $\bar{A}$ denotes the conjugate of $A$. Since $\operatorname{Tr} A=(\operatorname{tr} f(A)) / 2$, the result follows from the case when $\mathbf{F}=\mathbf{C}$.

Let $\mathbf{b}, \mathbf{c} \in \mathbf{R}^{n}$. We will write $\mathbf{b} \prec \mathbf{c}$ if $\mathbf{b} \in \mathcal{C}(\mathbf{c}, n)$. By the Hardy-LittlewoodPolya Theorem [HLP], [MO], we have $\mathbf{b} \prec \mathbf{c}$ if and only if

$$
\sum_{i=1}^{k} b_{[i]} \leq \sum_{i=1}^{k} c_{[i]} \quad(k=1, \ldots, n-1) \text { and } \sum_{i=1}^{n} b_{[i]}=\sum_{i=1}^{n} c_{[i]} .
$$

Lemma 2.6. For $\mathbf{c} \in \mathbf{R}^{n}$, let $\mathcal{U}_{\mathbf{F}}(\mathbf{c}, r)=\left\{U^{*}[\mathbf{b}] U: \mathbf{b} \in \mathcal{C}(\mathbf{c}, r), U \in \mathcal{U}_{n}(\mathbf{F})\right\}$. We have

a) For $1 \leq r \leq n$, conv $\mathcal{U}_{\mathbf{F}}(\mathbf{c}, r)=\left\{U^{*}[\mathbf{b}] U: \mathbf{b} \prec \mathbf{c}, U \in \mathcal{U}_{n}(\mathbf{F})\right\}=\mathcal{U}_{\mathbf{F}}(\mathbf{c}, n)$.

b) $\{\mathbf{b}: \mathbf{b} \prec \mathbf{c}\}=\left\{\operatorname{diag} U[\mathbf{c}] U^{*}: U \in \mathcal{U}_{n}(\mathbf{F})\right\}$ $=\left\{\operatorname{diag} U[\mathbf{b}] U^{*}: U \in \mathcal{U}_{n}(\mathbf{F}), \mathbf{b} \prec \mathbf{c}\right\}$.

c) $\mathcal{U}_{\mathbf{F}}(\mathbf{c}, n-1)$ has a convex boundary.

Proof. We may assume that $\mathbf{c}=\mathbf{c}_{\downarrow}$.

a) Since $\mathcal{U}_{\mathbf{F}}(\mathbf{c}, 1) \subseteq \cdots \subseteq \mathcal{U}_{\mathbf{F}}(\mathbf{c}, n)$, it suffices to prove that conv $\mathcal{U}_{\mathbf{F}}(\mathbf{c}, 1)=$ $\mathcal{U}_{\mathbf{F}}(\mathbf{c}, n)$. Thompson $[\mathrm{Th}]^{1}$ proved the result for $\mathbf{F}=\mathbf{C}$ and it was pointed out by Au-Yeung and Tsing [AT1] that the result also holds for $\mathbf{F}=\mathbf{R}$ and Q.

b) The first equality is due to Horn [Ho] and Au-Yeung and Tsing [AT1]. The second equality follows from the first equality.

c) Let $P_{\mathbf{c}}=\left\{A \in \mathcal{H}_{n}(\mathbf{F}): \operatorname{Tr} A=\sum_{i=1}^{n} c_{i}\right\}=\left\{[\mathbf{c}]+B: B \in \mathcal{H}_{n}(\mathbf{F}) \operatorname{tr} B=0\right\}$. Then $P_{\mathbf{c}}$ is a hyperplane containing $\mathcal{U}_{\mathbf{F}}(\mathbf{c}, n)$. From a), conv $\mathcal{U}_{\mathbf{F}}(\mathbf{c}, n-1)=$ $\mathcal{U}_{\mathbf{F}}(\mathbf{c}, n) \subseteq P_{\mathbf{c}}$. Suppose $B \in \mathcal{U}_{\mathbf{F}}(\mathbf{c}, n)$. Then there exists $U \in \mathcal{U}_{n}(\mathbf{F})$ and $\mathbf{b} \in \mathcal{C}(\mathbf{c}, n)$ such that $B=U[\mathbf{b}] U^{*}$. If equality holds in (1) for some $1 \leq$ $k<n$, then $B \in \mathcal{U}_{\mathbf{F}}(\mathbf{c}, n-1)$. Suppose strict inequality holds in (1) for all $1 \leq k<n$. Let $A \in P_{\mathbf{c}}$ have eigenvalues $\lambda_{1} \geq \cdots \geq \lambda_{n}$. If $A$ is sufficiently close to $B$ in $P_{\mathbf{c}}$, then we have $\sum_{i=1}^{k} \lambda_{i}<\sum_{i=1}^{k} c_{i}$ for $k=1, \ldots, n-1$. Hence, $A \in \mathcal{U}_{\mathbf{F}}(\mathbf{c}, n)$. Therefore the boundary of the convex set $\mathcal{U}_{\mathbf{F}}(\mathbf{c}, n)$ in $P_{\mathbf{c}}$ is contained in $\mathcal{U}_{\mathbf{F}}(\mathbf{c}, n-1)$.

Remark 2.7. Let $\mathbf{b} \in \mathbf{R}^{n}, A \in \mathcal{H}_{n}(\mathbf{F})$ and $U \in \mathcal{U}_{n}(\mathbf{F})$. It follows from Lemma 2.5 and $2.6 \mathbf{b}$ ) that $\operatorname{tr}[\mathbf{b}] U A U^{*}=\operatorname{Tr}[\mathbf{b}] U A U^{*}=\operatorname{Tr} U^{*}[\mathbf{b}] U A$. Thus we have

$$
W_{\mathbf{c}, r}^{\mathbf{F}}\left(A_{1}, \cdots, A_{p}\right)=\left\{\left(\operatorname{Tr} B A_{1}, \cdots, \operatorname{Tr} B A_{p}\right): B \in \mathcal{U}_{\mathbf{F}}(\mathbf{c}, r)\right\} \text {, and }
$$

$\operatorname{conv} W_{\mathbf{c}, r}^{\mathbf{F}}\left(A_{1}, \cdots, A_{p}\right)=W_{\mathbf{c}, n}^{\mathbf{F}}\left(A_{1}, \cdots, A_{p}\right)$ for all $1 \leq r \leq n$.

First, we prove a special case of Theorem 2.2.

Lemma 2.8. Let $p \leq f_{\mathbf{F}}(n)-2$. Then $W_{\mathbf{c}, n-1}^{\mathbf{F}}\left(A_{1}, \cdots, A_{p}\right)$ is convex for all $A_{1}, \cdots, A_{p} \in \mathcal{H}_{n}(\mathbf{F})$.

Proof. Let $\mathbf{w}=\left(w_{1}, \cdots, w_{p}\right) \in \operatorname{conv} W_{\mathbf{c}, n-1}^{\mathbf{F}}\left(A_{1}, \cdots, A_{p}\right)=W_{\mathbf{c}, n}^{\mathbf{F}}\left(A_{1}, \cdots, A_{p}\right)$. Then, by Remark 2.7, there exists $B \in \mathcal{U}_{\mathbf{F}}(\mathbf{c}, n)$ such that $w_{j}=\operatorname{Tr} B A_{j}$ for all $1 \leq j \leq p$. Since $p \leq f_{\mathbf{F}}(n)-2$, there exists a non-zero $X \in \mathcal{H}_{n}(\mathbf{F})$ such that $\operatorname{Tr} X=\operatorname{Tr} X A_{j}=0$ for all $1 \leq j \leq p$. For all $t \in \mathbf{R}$, we have $B+t X \in P_{\mathbf{c}}$ (as defined in the proof of Lemma $2.6 \mathrm{c})$ ) and $\operatorname{Tr}(B+t X) A_{j}=w_{j}$ for all $1 \leq j \leq p$. Since $\mathcal{U}_{\mathbf{F}}(\mathbf{c}, n)$ is bounded, we have, by Lemma $\left.2.6 \mathrm{c}\right), B+t X \in \mathcal{U}_{\mathbf{F}}(\mathbf{c}, n-1)$ for some $t$.

\footnotetext{
${ }^{1}$ The author is thankful to Professor Tin Yau Tam for pointing out this reference.
} 
Lemma 2.9. Let $p=f_{\mathbf{F}}(n)-1$ and $\mathcal{K}$ be the subspace spanned by $A_{1}, \cdots, A_{p}$ in $\mathcal{H}_{n}(\mathbf{F})$. Then we have

a) If $I_{n} \in \mathcal{K}$ or $\operatorname{dim} \mathcal{K}<p$, then $W_{\mathbf{c}, n-1}^{\mathbf{F}}\left(A_{1}, \cdots, A_{p}\right)$ is convex.

b) $W_{\mathbf{c}, n-1}^{\mathbf{F}}\left(A_{1}, \cdots, A_{p}\right)$ has a convex boundary.

Proof. a) Suppose $B_{1}, \cdots, B_{q}$ is a basis of $\mathcal{K}$. Then $W_{\mathbf{c}, n-1}^{\mathbf{F}}\left(A_{1}, \cdots, A_{p}\right)$ is convex if and only if $W_{\mathbf{c}, n-1}^{\mathbf{F}}\left(B_{1}, \cdots, B_{q}\right)$ is convex. If $q<p$, the result follows from Lemma 2.8.

Suppose $I_{n} \in \mathcal{K}$ and $q=p$. Then $A_{1}, \cdots, A_{p}$ is a basis of $\mathcal{K}$. We may assume that $A_{p}=I_{n}$. Since $\operatorname{Tr} X I_{n}=\sum_{i=1}^{n} c_{i}=s$ is independent of $X \in \mathcal{U}_{\mathbf{F}}(\mathbf{c}, n-1)$, we have $W_{\mathbf{c}, n-1}^{\mathbf{F}}\left(A_{1}, \cdots, A_{p}\right)=\left\{(\mathbf{w}, s): \mathbf{w} \in W_{\mathbf{c}, n-1}^{\mathbf{F}}\left(A_{1}, \cdots, A_{p-1}\right)\right\}$. Hence, the convexity of $W_{\mathbf{c}, n-1}^{\mathbf{F}}\left(A_{1}, \cdots, A_{p}\right)$ follows from that of $W_{\mathbf{c}, n-1}^{\mathbf{F}}\left(A_{1}, \cdots, A_{p-1}\right)$.

b) From a), we only need to consider the case when $\operatorname{dim} \mathcal{K}=p$ and $I_{n} \notin \mathcal{K}$; then $\left\{I_{n}, A_{1}, \cdots, A_{p}\right\}$ is a basis for $\mathcal{H}_{n}(\mathbf{F})$. We have

$$
W_{\mathbf{c}, n-1}^{\mathbf{F}}\left(A_{1}, \cdots, A_{p}\right) \cong W_{\mathbf{c}, n-1}^{\mathbf{F}}\left(A_{1}, \cdots, A_{p}, I_{n}\right) \cong \mathcal{U}_{\mathbf{F}}(\mathbf{c}, n-1) .
$$

Hence, by Lemma $2.6 \mathrm{c}), W_{\mathbf{c}, n-1}^{\mathbf{F}}\left(A_{1}, \cdots, A_{p}\right)$ has a convex boundary.

Let $\mathbf{b} \in \mathbf{R}^{n}$ and $1 \leq k \leq \ell \leq n$; we will denote $\left(b_{k}, \cdots, b_{\ell}\right)$ by $\mathbf{b}[k, \ell]$. Similarly, for $A=\left(a_{i, j}\right) \in \mathcal{M}_{n}(\mathbf{F}), A[k, \ell]$ will denote the principal submatrix $\left(a_{i j}\right)_{k \leq i, j \leq \ell}$ of $A$.

Lemma 2.10. Let $\mathcal{K}$ be a subspace of $\mathcal{H}_{n}(\mathbf{F})$ of dimension $f_{\mathbf{F}}(n-1)-1$. Then there exist a nonzero $A \in \mathcal{K}$ and $U \in \mathcal{U}_{n}(\mathbf{F})$ such that $\left(U A U^{*}\right)[1, n-1]=\alpha I_{n-1}$ for some $\alpha \in \mathbf{R}$. Moreover, $U$ can be chosen in the path connected component of $I_{n}$ in $\mathcal{U}_{n}(\mathbf{F})$.

Proof. If $I_{n} \in \mathcal{K}$, then we are done. Suppose $I_{n} \notin \mathcal{K}$. Let $\mathcal{K}_{1}$ be the subspace spanned by $I_{n}$ and $\mathcal{K}$. Let $\mathcal{L}=\mathcal{K}_{1}^{\perp}$ be the orthogonal complement of $\mathcal{K}_{1}$ in $\mathcal{H}_{n}(\mathbf{F})$, with respect to the inner product defined in Lemma 2.5. Let $m=\operatorname{dim} \mathcal{L}=$ $f_{\mathbf{F}}(n)-f_{\mathbf{F}}(n-1)$. By a result of Adams, Lax and Phillips ([ALP, Theorem 1]), there exists a nonzero singular matrix $B$ in $\mathcal{L}$. Choose $U \in \mathcal{U}_{n}(\mathbf{F})$ such that the last row and last column of $U B U^{*}$ are zero. Let $\mathcal{D}=\left\{D \in \mathcal{H}_{n}(\mathbf{F}): D[1, n-1]=0\right\}$. Since $U B U^{*} \in \mathcal{D}^{\perp} \cap\left(U \mathcal{L} U^{*}\right)$ and $\operatorname{dim} U \mathcal{L} U^{*}=\operatorname{dim} \mathcal{L}=\operatorname{dim} \mathcal{D}$, we have

$$
\begin{aligned}
& \operatorname{dim}\left(\mathcal{D} \cap\left(U \mathcal{L} U^{*}\right)^{\perp}\right) \\
= & \operatorname{dim} \mathcal{D}+\operatorname{dim}\left(U \mathcal{L} U^{*}\right)^{\perp}-\operatorname{dim}\left(D+\left(U \mathcal{L} U^{*}\right)^{\perp}\right) \\
= & f_{\mathbf{F}}(n)-\operatorname{dim}\left(D+\left(U \mathcal{L} U^{*}\right)^{\perp}\right) \\
= & \operatorname{dim}\left(\left(D+\left(U \mathcal{L} U^{*}\right)^{\perp}\right)^{\perp}\right)=\operatorname{dim}\left(\mathcal{D}^{\perp} \cap\left(U \mathcal{L} U^{*}\right)\right)>0 .
\end{aligned}
$$

Let $D$ be a nonzero matrix in $\mathcal{D} \cap\left(U \mathcal{L} U^{*}\right)^{\perp}=\mathcal{D} \cap U \mathcal{K}_{1} U^{*}$. Then there exist $A \in \mathcal{K}$ and $\alpha \in \mathbf{R}$ such that $U\left(A-\alpha I_{n}\right) U^{*}=D \in \mathcal{D}$. Thus, $\left(U A U^{*}\right)[1, n-1]=\alpha I_{n-1}$. If $A=0$, then $\alpha=0$ because $D[1, n-1]=0$. This would imply $D=0$, a contradiction. Hence, we have $A \neq 0$.

By Lemma $2.4 \mathrm{~b}), U$ is in the connected component of $I_{n}$ in $\mathcal{U}_{n}(\mathbf{F})$ for $\mathbf{F}=\mathbf{C}$ and $\mathbf{Q}$. For $\mathbf{F}=\mathbf{R}$, by multiplying $U$ by $I_{n-1} \oplus[-1]$, if necessary, we get $\operatorname{det} U=1$ and the result follows.

Remark 2.11. Suppose $A \in \mathcal{H}_{n}(\mathbf{F})$ has eigenvalues $\lambda_{1} \geq \cdots \geq \lambda_{n}$ and $\alpha \in \mathbf{R}$. Then direct computation shows that $\lambda_{2}=\cdots=\lambda_{n-1}=\alpha$ if and only if there 
exists $U \in \mathcal{U}_{n}(\mathbf{F})$ such that $U A U^{*}[1, n-1]=\alpha I_{n-1}$. Therefore Lemma 2.10 can be restated as follows.

Proposition 2.12. Let $\mathcal{K}$ be a subspace of $\mathcal{H}_{n}(\mathbf{F})$ of dimension $f_{\mathbf{F}}(n-1)-1$. Then there exists a nonzero $A \in \mathcal{K}$ such that $\lambda_{2}(A)=\cdots=\lambda_{n-1}(A)$.

It would be interesting to see if there is any direct relationship between Propositions 1.3 and 2.12 .

The following lemma is an extension of an idea contained in a paper by $\mathrm{Au}$-Yeung and Tsing [AT2].

Lemma 2.13. Let $r=n-2 \geq 1, p=f_{\mathbf{F}}(r+1)-1$ and $A_{1}, \cdots, A_{p} \in \mathcal{H}_{n}(\mathbf{F})$. Suppose $\mathbf{c}=\mathbf{c}_{\downarrow} \in \mathbf{R}^{n-1}, \mathbf{b} \in \mathcal{C}(\mathbf{c}, n-1)$ and $e \in \mathbf{R}$ such that either $e \leq c_{n-1}$ or $e \geq c_{1}$. Then there exist $\mathbf{d} \in \mathcal{C}(\mathbf{c}, r)$ and $U \in \mathcal{U}_{n}(\mathbf{F})$ such that $\operatorname{Tr}([\mathbf{b}] \oplus[e]) A_{j}=$ $\operatorname{Tr}([\mathbf{d}] \oplus[e]) U A_{j} U^{*}$ for all $1 \leq j \leq p$.

Proof. Let $\mathcal{K}$ be the subspace spanned by $A_{1}, \cdots, A_{p}$. If $\operatorname{dim} \mathcal{K}<p$, let $B_{i}=$ $A_{i}[1, n-1]$ for $1 \leq i \leq p$. Then, by Lemma $\left.2.9 \mathrm{a}\right), W_{\mathbf{c}, r}^{\mathbf{F}}\left(B_{1}, \cdots, B_{p}\right)$ is convex and the result follows.

Suppose $\operatorname{dim} \mathcal{K}=p$. Then by Lemma 2.10 , we can get $U_{1}$ in the path connected component of $I_{n}$ in $\mathcal{U}_{n}(\mathbf{F})$ such that $\left(U_{1} A U_{1}^{*}\right)[1, n-1]=\alpha I_{n-1}$ for some nonzero $A \in \mathcal{K}$ and $\alpha \in \mathbf{R}$.

Let $U(t), 0 \leq t \leq 1$, be a path in $\mathcal{U}_{n}(\mathbf{F})$ such that $U(0)=I_{n}$ and $U(1)=U_{1}$. For every $0 \leq t \leq 1$ and $1 \leq j \leq p$, let $\left(U(t) A_{j} U(t)^{*}\right)[1, n-1]=B_{j}(t)$ and $\left(U(t) A_{j} U(t)^{*}\right)[n, n]=a_{j}(t)$. Define

$$
W(t)=W_{\mathbf{c}, r}^{\mathbf{F}}\left(B_{1}(t), \cdots, B_{p}(t)\right)+\left\{e\left(a_{1}(t), \cdots, a_{p}(t)\right)\right\} .
$$

Then $W(t)$ varies continuously with $t$. Let $\mathcal{K}_{1}$ be the subspace of $\mathcal{H}_{n-1}(\mathbf{F})$ spanned by $B_{1}(1), \cdots, B_{p}(1)$. Then either $I_{n-1} \in \mathcal{K}_{1}$ or $\operatorname{dim} \mathcal{K}_{1}<p$. We have

a) $\mathbf{q}=\left(\operatorname{Tr}([\mathbf{b}] \oplus[e]) A_{j}\right)_{1<j<p} \in \operatorname{conv} W(0)$.

b) For each $0 \leq t \leq 1, W(t)$ has a convex boundary and is contained in

$$
\left\{\left(\operatorname{Tr}([\mathbf{d}] \oplus[e]) U A_{j} U^{*}\right)_{1 \leq j \leq p}: \mathbf{d} \in \mathcal{C}(\mathbf{c}, r), U \in \mathcal{U}_{n}(\mathbf{F})\right\} .
$$

c) $W(1)$ is convex.

If $\mathbf{q} \in W(0)$, then the result follows from b). Suppose $W(0)$ is not convex and $\mathbf{q}$ lies in the interior of conv $W(0)$.

Since $W(t)$ varies continuously with $t$ and each $W(t)$ has a convex boundary, we have either 1) $W(t)$ is convex for some $t>0$ and $\mathbf{q} \in W(t)$ or 2) there exists $t<1$ such that $\mathbf{q}$ lies on the boundary of conv $W(t)$. In both cases, the result follows from b).

Proof of Theorem 2.2. For $r=n-1$, the result follows from Lemma 2.8. So, we may assume that $1 \leq r \leq n-2$. Therefore, $2 \leq r+1 \leq n-1$ and $p \leq f_{\mathbf{F}}(r+1)-1$.

Suppose $\mathbf{w} \in W_{\mathbf{c}, n}^{\overline{\mathbf{F}}}\left(A_{1}, \cdots, A_{p}\right)$. Let $s=\min \left\{i: \mathbf{w} \in W_{\mathbf{c}, i}^{\mathbf{F}}\left(A_{1}, \cdots, A_{p}\right)\right\}$. It suffices to show that $s \leq r$. Let $\mathbf{w}=\left(w_{1}, \cdots, w_{p}\right)$. There exist $\mathbf{b} \in \mathcal{C}(\mathbf{c}, s)$ and $U \in \mathcal{U}_{n}(\mathbf{F})$ such that $w_{j}=\operatorname{Tr}[\mathbf{b}] U A_{j} U^{*}$ for all $1 \leq j \leq p$. Moreover, there exist $D_{i} \in \Omega\left(n_{i}\right)$ with $n_{i} \leq s$ for $1 \leq i \leq m$ and $\sum_{i=1}^{m} n_{i}=n$ such that $\mathbf{b}=\mathbf{c}\left(\oplus_{i=1}^{m} D_{i}\right)$. Let $k_{0}=0, k_{i}=\sum_{j=1}^{i} n_{j}, \mathbf{c}^{\mathbf{i}}=\mathbf{c}\left[k_{i-1}+1, k_{i}\right]$ and $\mathbf{b}^{\mathbf{i}}=\mathbf{b}\left[k_{i-1}+1, k_{i}\right]$ for $1 \leq i \leq m$. Let $\mathcal{A}_{j}^{i}=\left(U A_{j} U^{*}\right)\left[k_{i-1}+1, k_{i}\right]$ for $1 \leq j \leq p$. Then $w_{j}=\sum_{i=1}^{m} \operatorname{Tr}\left[\mathbf{b}^{\mathbf{i}}\right] \mathcal{A}_{j}^{i}$, for all $1 \leq j \leq p$. 
If $n_{i}>r+1$ for some $i$, then it follows from $p \leq f_{\mathbf{F}}(r+2)-2$ and Lemma 2.8 that there exist $\mathbf{d}^{\mathbf{i}} \in \mathcal{C}\left(\mathbf{c}^{\mathbf{i}}, r+1\right)$ and $U_{i} \in \mathcal{U}_{n_{i}}(\mathbf{F})$ such that $\operatorname{Tr}\left[\mathbf{b}^{\mathbf{i}}\right] \mathcal{A}_{j}^{i}=\operatorname{Tr}\left[\mathbf{d}^{\mathbf{i}}\right] U_{i} \mathcal{A}_{j}^{i} U_{i}^{*}$ for all $1 \leq j \leq p$. Hence we may assume that $s \leq r+1$. Suppose $n_{i}=r+1$. If $p \leq f_{\mathbf{F}}(r+1)-2$, then by Lemma $2.8, W_{\mathbf{c}^{i}, n_{i}}^{\mathbf{F}}\left(\mathcal{A}_{1}^{i}, \cdots, \mathcal{A}_{p}^{i}\right)$ is convex. If $p=$ $f_{\mathbf{F}}(r+1)-1$, then we can apply Lemma 2.13 to $\mathbf{b}^{\mathbf{i}}, \mathbf{c}^{\mathbf{i}}$ and $\left(U A U^{*}\right)\left[k_{i-1}+1, k_{i}+1\right]$ if $i<m$ (or $\left(U A U^{*}\right)\left[k_{i-1}, k_{i}\right]$ if $i=m$ ). In all cases, we can get $\mathbf{d}^{\mathbf{i}} \in \mathcal{C}\left(\mathbf{c}^{\mathbf{i}}, r\right)$, $\mathbf{d}=\left(\mathbf{b}^{\mathbf{1}}, \cdots, \mathbf{b}^{\mathbf{i}-\mathbf{1}}, \mathbf{d}^{\mathbf{i}}, \mathbf{b}^{\mathbf{i}+\mathbf{1}} \cdots, \mathbf{b}^{\mathbf{m}}\right)$ and $V \in \mathcal{U}_{n}(\mathbf{F})$ such that $w_{j}=\operatorname{Tr}[\mathbf{d}] V A_{j} V^{*}$ for all $1 \leq j \leq p$. Repeating this procedure, we get $s \leq r$.

\section{SOME RELATED RESUlts}

Proposition 3.1. Let $\mathbf{F}=\mathbf{R}, \mathbf{C}$ or $\mathbf{Q}$ and $A_{1}, \cdots, A_{p} \in \mathcal{H}_{n}(\mathbf{F})$. Then for every $\mathbf{c}=\left(c_{1}, \cdots, c_{n}\right) \in \mathbf{R}^{n}$ and $1 \leq r \leq n$, the following conditions are equivalent:

a) $W_{\mathbf{c}, r}^{\mathbf{F}}\left(A_{1}, \cdots, A_{p}\right)$ is convex.

b) If $\left(r_{1}, \cdots, r_{p}\right) \in \mathbf{R}^{p} \backslash W_{\mathbf{c}, r}^{\mathbf{F}}\left(A_{1}, \cdots, A_{p}\right)$, then there exist $\alpha_{1}, \cdots, \alpha_{p} \in \mathbf{R}$ such that

$$
\operatorname{Tr} X\left(\sum_{j=1}^{p} \alpha_{j} A_{j}\right)>\sum_{j=1}^{p} \alpha_{j} r_{j} \text {, for all } X \in \mathcal{U}_{\mathbf{F}}(\mathbf{c}, n) .
$$

c) If $\left(r_{1}, \cdots, r_{p}\right) \in \mathbf{R}^{p} \backslash W_{\mathbf{c}, r}^{\mathbf{F}}\left(A_{1}, \cdots, A_{p}\right)$, then there exist $\alpha_{1}, \cdots, \alpha_{p} \in \mathbf{R}$ such that the eigenvalues of $A=\sum_{j=1}^{p} \alpha_{j} A_{j}$ satisfies $\sum_{i=1}^{n} c_{\boldsymbol{\sigma}(i)} \lambda_{i}(A)>\sum_{j=1}^{p} \alpha_{j} r_{j}$, for all permutation $\sigma$ of $\{1, \cdots, n\}$.

d) $W_{\mathbf{b}, r}^{\mathbf{F}}\left(A_{1}, \cdots, A_{p}\right) \subseteq W_{\mathbf{c}, r}^{\mathbf{F}}\left(A_{1}, \cdots, A_{p}\right)$ for all $\mathbf{b} \prec \mathbf{c}$.

Some special cases of Proposition 3.1 are given by Au-Yeung and Tsing in [AT1] (for $p=2$ and $r=1$ ) and [AT2] (for $\mathbf{F}=\mathbf{C}$ and $r=1$ ). The proof of the general case is similar to the one given in [AT1]. Also, see [A2] for some related results.

Suppose $1 \leq r \leq n-1$ and $p<f_{\mathbf{F}}(r+1)-\delta_{n, r+1}$. It follows from Theorem 2.2 that all the conditions in Proposition 3.1 hold. In particular, by putting $\left(r_{1}, \cdots, r_{p}\right)=(0, \cdots, 0)$ in Proposition $\left.3.1 \mathrm{c}\right)$, we have the following generalization of Proposition 1.2.

Theorem 3.2. Let $\mathbf{F}=\mathbf{R}, \mathbf{C}$ or $\mathbf{Q}, 1 \leq r \leq n-1, p<f_{\mathbf{F}}(r+1)-\delta_{n, r+1}$ and $A_{1}, \cdots, A_{p} \in \mathcal{H}_{n}(\mathbf{F})$. Suppose $(0, \cdots, 0) \notin W_{\mathbf{c}, r}^{\mathbf{F}}\left(A_{1}, \cdots, A_{p}\right)$. Then there exist $\alpha_{1}, \cdots, \alpha_{p} \in \mathbf{R}$ such that the eigenvalues of $A=\sum_{j=1}^{p} \alpha_{j} A_{j}$ satisfy $\sum_{i=1}^{n} c_{\boldsymbol{\sigma}(i)} \lambda_{i}(A)>0$ for every permutation $\boldsymbol{\sigma}$ of $\{1, \cdots, n\}$.

From Theorem 3.2, we have the following generalization of Proposition 1.3.

Theorem 3.3. Let $\mathbf{F}=\mathbf{R}, \mathbf{C}$ or $\mathbf{Q}, 1<r<n$, and $\mathbf{k}(r)=f_{\mathbf{F}}(n)-f_{\mathbf{F}}(n+1-r)$. Suppose $\mathcal{K}$ be a subspace of $\mathcal{H}_{n}(\mathbf{F})$ with dimension $\geq \mathbf{k}(r)$. Then for every $\mathbf{c} \in \mathbf{R}^{n}$, with $c_{i} \neq c_{j}$ for some $i, j$, there exists a nonzero matrix $A \in \mathcal{K}$ such that $A=\lambda I+t B$ for some $\lambda, t \in \mathbf{R}$ and $B \in \mathcal{U}_{\mathbf{F}}(\mathbf{c}, n-r)$.

Proof. Without loss of generality, we may assume $c_{1} \geq \cdots \geq c_{n}>0$. Let $\mathcal{K}$ be a subspace of $\mathcal{H}_{n}(\mathbf{F})$ with dimension $\geq \mathbf{k}(r)=f_{\mathbf{F}}(n)-f_{\mathbf{F}}(n+1-r)$. Suppose the contrary that for every nonzero $A \in \mathcal{K}$, we have $A \neq \lambda I_{n}+t B$ for all $\lambda, t \in \mathbf{R}$ and $B \in \mathcal{U}_{\mathbf{F}}(\mathbf{c}, n-r)$. Then 1) $I_{n} \notin \mathcal{K}$ and 2) $\mathcal{K}_{1} \cap \mathcal{U}_{\mathbf{F}}(\mathbf{c}, n-r)=\emptyset$, where $\mathcal{K}_{1}$ is the subspace spanned by $\mathcal{K}$ and $I_{n}$. Let $\mathcal{L}=\mathcal{K}_{1}^{\perp}$. It follows from 1 ) that 
$\operatorname{dim} \mathcal{L}=p<f_{\mathbf{F}}(n+1-r)$. Since $1<r<n$, we have $0<n-r<n-1$. Let $\left\{A_{1}, \cdots, A_{p}\right\}$ be a basis of $\mathcal{L}$. 2) implies that $(0, \cdots, 0) \notin W_{\mathbf{c}, n-r}^{\mathbf{F}}\left(A_{1}, \cdots, A_{p}\right)$. Hence, by Theorem 3.2, there exists $A \in \mathcal{L}$ such that $\sum_{i=1}^{n} c_{\boldsymbol{\sigma}(i)} \lambda_{i}(A)>0$ for every permutation $\boldsymbol{\sigma}$ of $\{1, \cdots, n\}$. This implies that $\operatorname{Tr} A=\sum_{i=1}^{n} \lambda_{i}(A)>0$ because $\sum_{i=1}^{n} c_{i}>0$. But $I_{n} \in \mathcal{K}_{1}=\mathcal{L}^{\perp}$. So we have $\operatorname{Tr} A=\operatorname{Tr} A I_{n}=0$, a contradiction.

As we have pointed out in Remark 1.4 a), the bound $f_{\mathbf{F}}(r+1)-\delta_{n, r+1}$ is best possible for Proposition 1.1. The following theorem is an extension of this result.

Theorem 3.4. Suppose $c_{1} \geq \cdots \geq c_{n}$. If $c_{1}>c_{r+1}$ or $c_{n-r}>c_{n}$ and $p \geq$ $f_{\mathbf{F}}(r+1)-\delta_{n, r+1}$, then there exist $A_{1}, \cdots, A_{p} \in \mathcal{H}_{n}(\mathbf{F})$ such that $W_{\mathbf{c}, r}^{\mathbf{F}}\left(A_{1}, \cdots, A_{p}\right)$ is not convex.

Proof. It suffices to prove the case when $p=f_{\mathbf{F}}(r+1)-\delta_{n, r+1}$.

We may assume that $c_{1}>c_{r+1} \geq c_{n}>0$. Let $d=\left(\sum_{i=1}^{r+1} c_{i}\right)(r+1)^{-1}$ and $s=\sum_{i=1}^{n} c_{i}$. Let $\mathcal{K}=\left\{A \in \mathcal{H}_{n}(\mathbf{F}): A[1, r+1]=\lambda I_{r+1}\right.$ and $\left.d \operatorname{tr} A=\lambda s\right\}$. Then $\mathcal{K}$ has dimension $f_{\mathbf{F}}(n)-p$. Let $A_{1}, \cdots, A_{p} \in \mathcal{H}_{n}(\mathbf{F})$ be a basis of $\mathcal{K}^{\perp}$. Since $d I_{r+1} \oplus[\mathbf{c}[r+2, n]] \in \mathcal{K} \cap \mathcal{U}_{\mathbf{F}}(\mathbf{c}, r+1)$, we have $(0, \cdots, 0) \in W_{\mathbf{c}, r+1}^{\mathbf{F}}\left(A_{1}, \cdots, A_{p}\right)$. We are going to show that $(0, \cdots, 0) \notin W_{\mathbf{c}, r}^{\mathbf{F}}\left(A_{1}, \cdots, A_{p}\right)$. So, $W_{\mathbf{c}, r}^{\mathbf{F}}\left(A_{1}, \cdots, A_{p}\right)$ is not convex.

Suppose $(0, \cdots, 0) \in W_{\mathbf{c}, r}^{\mathbf{F}}\left(A_{1}, \cdots, A_{p}\right)$. Then there exists $A=\left(a_{i j}\right) \in \mathcal{K} \cap$ $\mathcal{U}_{\mathbf{F}}(\mathbf{c}, r)$. We have $a_{i i}=d$ for $1 \leq i \leq r+1$. By Lemma $\left.2.6 \mathrm{~b}\right)$, we have

$$
\sum_{i=1}^{r+1} c_{i}=\sum_{i=1}^{r+1} a_{i i} \leq \sum_{i=1}^{r+1} \lambda_{i}(A) \leq \sum_{i=1}^{r+1} c_{i} .
$$

Hence, $\sum_{i=1}^{r+1} a_{i i}=\sum_{i=1}^{r+1} \lambda_{i}(A)$. By a result of Li [Li, Lemma 4.1 ], $a_{i j}=a_{j i}=0$ for all $1 \leq i \leq r+1$ and $r+2 \leq j \leq n$. This implies that $\lambda_{i}(A)=d$ for $1 \leq i \leq r+1$. Since $A \in \mathcal{U}_{\mathbf{F}}(\mathbf{c}, r)$, we have $\sum_{i=1}^{m} d=\sum_{i=1}^{m} c_{i}$ for some $1 \leq m \leq r$. This gives

$$
\begin{aligned}
& \sum_{i=1}^{m} \sum_{j=1}^{r+1} c_{j}=\sum_{j=1}^{r+1} \sum_{i=1}^{m} c_{i} \\
\Rightarrow & \sum_{i=1}^{m} \sum_{j=m+1}^{r+1} c_{j}=\sum_{j=m+1}^{r+1} \sum_{i=1}^{m} c_{i} \\
\Rightarrow & \sum_{i=1}^{m} \sum_{j=m+1}^{r+1}\left(c_{i}-c_{j}\right)=0 \\
\Rightarrow & c_{1}=c_{r+1},
\end{aligned}
$$

a contradiction.

\section{REFERENCES}

[ALP] J. F. Adams, P. D. Lax and R. S. Phillips, On matrices whose real linear combinations are nonsingular, Proc. Amer. Math. Soc. 16 (1965), 318-322. MR 31:3432

[A1] Y. H. Au-Yeung, A simple proof of the convexity of the field of values defined by two hermitian forms, Aequations Math. 12 (1975), 82-83. MR 51:3200

[A2] - Some results on generalized numerical ranges and related topics, Current trends in matrix theory (F. Uhlig and R. Grone, eds.), North-Holland, New York, 1987, pp. (23-28). MR 88f: 15041 
[AP] Y. H. Au-Yeung and Y. T. Poon, A remark on the convexity and positive definiteness concerning hermitian matrices, Southeast Asian Bull. Math. 3 (1979), 85-92. MR 81c:15026

[AT1] Y. H. Au-Yeung and N. K. Tsing, Some theorems on the generalized numerical ranges, Linear and Multilinear Algebra 15 (1984), 3-11. MR 85c:15039

[AT2] - An extension of the Hausdorff-Toeplitz theorem on the numerical range, Proc. Amer. Math. Soc. 89 (1983), 215-218. MR 85f:15021

[Be] C. A. Berger, Normal dilations, Ph. D. Thesis, Cornell Univ., 1963.

[Bo] F. Bohnenblust, Joint positiveness of matrices, unpublished manuscript.

[Br] L. Brickman, On the field of values of a matrix, Proc. Amer. Math. Soc. 12 (1961), 61-66. MR 23:A160

[D] Doyle, Analysis of feedback systems with structured uncertainties, Proc. IEEE-D 129 (1982), 242-250. MR 84a:93035

[FT] M. K. H. Fan and A. L. Tits, m-form numerical range and the computation of the structured singular value, IEEE Trans. Automat. Control 33 (1988), 284-289. MR 89b:93051

[FL] S. Friedland and R. Loewy, Subspaces of symmetric matrices containing matrices with a multiple first eigenvalue, Pacific J. Math. 62 (1976), 389-399. MR 54:2698

[Hal1] P. Halmos, Numerical ranges and normal dialtions, Acta Sci. Math. (Szeged) 25 (1964), 1-5. MR 30:1399

[Hal2] _ _ A Hilbert space problem book, Von Nostrand, New York, 1967. MR 34:8178

[HLP] G. H. Hardy, J. E. Littlewood and G. Pólya, Inequalities, Cambridge University Press, London, 1934.

[Hau] F. Hausdorff, Der Wertvorrat einer Bilinearform, Math Z. 3 (1919), 314-316.

[Ho] A. Horn, Doubly stochastic matrices and the diagonal of a rotation matrix, Amer. J. Math. 76 (1954), 620-630. MR 16:105c

[HJ] R. A. Horn and C. A. Johnson, Matrix Analysis, Cambridge University Press, New York, 1985. MR 87e: 15001

[Le] H. C. Lee, Eigenvalues and canonical forms of matrices with quaternion coefficients, Proc. Roy. Irish. Acad. Sect. A 52 (1949), 253-260. MR 12:153i

[Li] C. K. Li, Matrices with some extremal properties, Linear Algebra Appl. 101 (1988), 255267. MR 89c: 15025

[MO] A. W. Marshall and I. Olkin, Inequalities: Theory of majorization and its applications, Academic Press, New York, 1979. MR 81b:00002

[P1] Y. T. Poon, Another proof of a result of Westwick, Linear and Multilinear Algebra 9 (1980), 35-37. MR 81h:15015

[P2] - On the convex hull of the multiform numerical range, Linear and Multilinear Algebra 37 (1994), 221-223. MR 95j: 15022

[Ta] O. Taussky, Positive definite matrices, Inequalities I (O. Shisha, eds.), Academic Press, New York, 1965. MR 36:3806

[Th] R. C. Thompson, Singular values, diagonal elements and convexity, SIAM J. Appl. Math. 32 (1977), 39-63. MR 54:12805

[To] O. Toeplitz, Das algebraishe Analogon zu einem Satze von Fejér, Math. Z. 2 (1918), 187197.

[W] R. Westwick, A theorem on numerical range, Linear and Multilinear Algebra 2 (1975), 311-315. MR 51:11132

Department of Mathematics, Iowa State University, Ames, Iowa 50011

E-mail address: ytpoon@iastate.edu 